\title{
An automatic analysis method for detecting and eliminating ECG artifacts in EEG
}

\author{
Joe-Air Jiang ${ }^{\mathrm{a}}$, Chih-Feng Chao ${ }^{\mathrm{a}}$, Ming-Jang Chiu ${ }^{\mathrm{b}}$, Ren-Guey Lee ${ }^{\mathrm{c}, *}$, \\ Chwan-Lu Tseng ${ }^{\mathrm{d}}$, Robert Lin $^{\mathrm{a}}$ \\ ${ }^{a}$ Department of Bio-Industrial Mechatronics Engineering, National Taiwan University, Taipei, Taiwan \\ ${ }^{\mathrm{b}}$ Department of Neurology, National Taiwan University Hospital, Taipei, Taiwan \\ ${ }^{\mathrm{c}}$ Department of Electronic Engineering, National Taipei University of Technology, Taipei 10643, Taiwan \\ ${ }^{\mathrm{d}}$ Department of Electric Engineering, National Taipei University of Technology, Taipei, Taiwan
}

Received 21 January 2006; received in revised form 25 March 2007; accepted 29 March 2007

\begin{abstract}
An automated method for detecting and eliminating electrocardiograph (ECG) artifacts from electroencephalography (EEG) without an additional synchronous ECG channel is proposed in this paper. Considering the properties of wavelet filters and the relationship between wavelet basis and characteristics of ECG artifacts, the concepts for selecting a suitable wavelet basis and scales used in the process are developed. The analysis via the selected basis is without suffering time shift for decomposition and detection/elimination procedures after wavelet transformation. The detection rates, above $97.5 \%$ for MIT/BIH and NTUH recordings, show a pretty good performance in ECG artifact detection and elimination.
\end{abstract}

(C) 2007 Elsevier Ltd. All rights reserved.

Keywords: EEG rhythms; ECG artifacts; Wavelet basis selection criterion; Wavelet transform

\section{Introduction}

The electroencephalography (EEG) represents the excitatory and inhibitory postsynaptic potentials generated by cortical nerve cells. EEGs enable brain functions to be investigated via monitoring potential waves from the scalp surface. Since EEGs were discovered by Berger in 1929, they have been considered an important tool for diagnosing, monitoring, and managing neurological states in clinical medicine. Nowadays, EEG measurement is widely used as a standard procedure in research including sleep studies [1], epileptic abnormalities [2-4], and the diagnosis of other disorders [5,6]. However, inspecting large quantities of raw EEG records visually is a complex and time-consuming operation. Visual screening of EEG records requires an experienced professionals - the electroencephalographer (EEGer). When understanding EEGs to identify

\footnotetext{
* Corresponding author. Tel.: +88622771 2171x2254

E-mail address: evans@ntut.edu.tw (R.-G. Lee).
}

specific conditions such as epilepsy or sleep stages, an EEGer is guided by general definitions and subjective criteria based on the causal relationship of extended EEG. Large quantities of reviewing records, covering more than several hours or even a day, are normally required to diagnosis disorders or determine sleep stages. Since artifacts may cause inaccuracy or even critical errors during visually screening or computer analysis, pure EEG records without artifacts will improve the accuracy of visual inspection or computer-based analysis in the long term.

In general, the relatively high electrical energy cardiac activity is most likely to cause EEG artifacts. Electrocardiograph (ECG) artifacts remain a major problem for EEG research. Some automatic detection methods have been proposed in past studies [7,8]. Algorithms for eliminating artifacts have been developed using the ensemble average subtraction (EAS) method [7], independent component analysis (ICA), and adaptive noise canceling theory [8]. Nevertheless, these methods cannot be implemented without an additional reference ECG channel, and the ECG signal is therefore required to use these algorithms. 
Recently, an energy interval histogram method based on the Smoothed Nonlinear Energy Operator (SNEO) technique and multiple thresholding schemes was proposed by Park and Jeong [8]. The SNEO technique is known to be very sensitive to noise, and EEGs contain abundant noise such as EMG artifacts and other high frequency disturbances. As a result, several postprocessing procedures are needed to complete detection and elimination of artifacts after SNEO processing. The total failed detection rate for four subjects was $9.1 \%$ under restricted conditions. Detection and elimination algorithms are thus worthy of further investigation for the sake of the improvement of performance.

The wavelet transform (WT) method has various uses in biomedical applications [9]. Seizure detection in epilepsy patients is one of the most frequent uses of WT in EEG analysis $[2-4,10]$. WT is also used as a pre-processing or feature extraction tool prior classification and clustering during EEG analysis. Kalayci and Ozdamar used an artificial neural network to discriminate between epileptic spikes and background EEG activities, and chose to employ Daub-2 and Daub-10 as a wavelet basis [3]. Shen and Sun also used Daub-2 as a basis, and proposed a method to extract dynamic rhythms via MultiResolution Analysis (MRA) such that four primary rhythms were acquired individually and reconstructed by decomposition of the raw EEG data [11]. It is obvious that the Daub- $N$ basis has been frequently applied [3,4,6,10,11]. However, applying MRA with a Daub- $N$ basis to decompose/extract ECG artifacts entails certain problems.

This paper presents a novel method of detecting and eliminating ECG artifacts based on the WT technique. In contrast to the foregoing studies, the proposed method takes advantage of the WT technique's localization and provides more temporal accuracy when performing long-term detection and elimination directly from EEGs. In other words, our algorithm does not need an additional synchronous ECG channel to perform detection and elimination. Our ECG artifact detection and elimination procedures are described in Section 2. Section 3 provides illustrations of detection and elimination schemes for both single and multi-channel cases.

\section{Methods}

\subsection{Wavelet transform}

The WT of signal $x(t)$ is defined as

$C(a, b)=\int_{R} x(t) \psi_{a, b}(t) \mathrm{d} t=\int_{R} x(t) \frac{1}{\sqrt{a}} \psi\left(\frac{t-b}{a}\right) \mathrm{d} t$.

Here $C(a, b)$ are the WT coefficients of signal $x(t), \psi(\cdot)$ is the wavelet function, $a$ is a scale parameter, and $b$ is a translation parameter. $a \in R^{+}-\{0\}, b \in R$ for continuous analysis; $a=2^{j}, b=k 2^{j}$, and $(j, k) \in Z^{2}$ for discrete analysis. $j$ is the decomposition level. Since the continuous wavelet transform (CWT) method is capable of performing continuous translation and is not restricted to a dyadic scale, it provides a higher resolution with a continuous scale for dilation. This property of
CWT makes it possible to localize features in both time and frequency domains for accurate detection. In many situations, however, the discrete wavelet transform (DWT) method is sufficient to satisfy the requirements of analysis. The DWT method is a dyadic WT when the scaling parameter $a$ is a number with a power of two, i.e., $a=2^{j}, j \in Z$. Mallat's fast algorithm for DWT is computed as follows [12]:

$$
\begin{aligned}
& A_{j}(n)=\sum_{k=-\infty}^{+\infty} g(k-2 n) A_{j-1}(k), \\
& D_{j}(n)=\sum_{k=-\infty}^{+\infty} h(k-2 n) A_{j-1}(k) .
\end{aligned}
$$

Here $A_{j}$ is termed the $j$ th approximation, or approximation at level $j$; and $D_{j}$ is termed the $j$ th detail, or detail at level $j$. The filters $h(\cdot)$ and $g(\cdot)$ used to decompose signals are called wavelet filters (high-pass) and scaling filters (lowpass), respectively. Transformation via these filters is known as MRA and is used to decompose signals into sub-bands with scale-dependent bandwidths [12]; the time-dependent spectral features of physiological signals can then be decomposed into individual levels. Therefore, dyadic WT can be considered to yield a set of equivalent filter-banks generated by the original wavelet functions via spectral analysis. When the decomposition level is set as $m$, the raw EEG is filtered into each level by filter-banks $H_{1}(\omega), H_{2}(\omega), \ldots, H_{m}(\omega)$ and $G_{1}(\omega), G_{2}(\omega), \ldots, G_{m}(\omega)$, where $H_{j}(\omega)$ and $G_{j}(\omega)$ at each decomposition level $j$ are defined as follows [13]:

$$
\left\{\begin{array}{l}
G_{j}(\omega)=\prod_{l=0}^{j-1} G\left(2^{l} \omega\right), \\
H_{j}(\omega)=H\left(2^{j-1} \omega\right) G_{j-1}(\omega) .
\end{array}\right.
$$

The band of each filter at each decomposition level can thus be obtained. For example, the multi-band frequency of wavelet basis, Coiflet-1, is shown in Fig. 1. The main frequency band of each level can be roughly defined as $\left(f_{\mathrm{s}} / 2^{j-1}\right) \leqslant\left\|H_{j}\right\| \leqslant\left(f_{\mathrm{s}} / 2^{j}\right)$, which implies that the bandwidth at the current level is approximately half of that at the previous level.

\subsection{Wavelet basis and scale selection}

Choosing a suitable wavelet basis for EEG analysis relies on three aspects: the temporal morphology (pattern) relationship between the EEG and the wavelet basis, the frequency band allocation relationship between the EEG and the wavelet basis, and the properties of the wavelet filters.

As for the aspect of frequency, WT decomposes a signal into several bands, of which some specific bands will contain the critical features of an EEG. As a consequence, the overriding consideration is to choose a suitable decomposition level/scale corresponding to the bands containing critical features. As for morphological aspects, choosing a basis with a similar temporal 

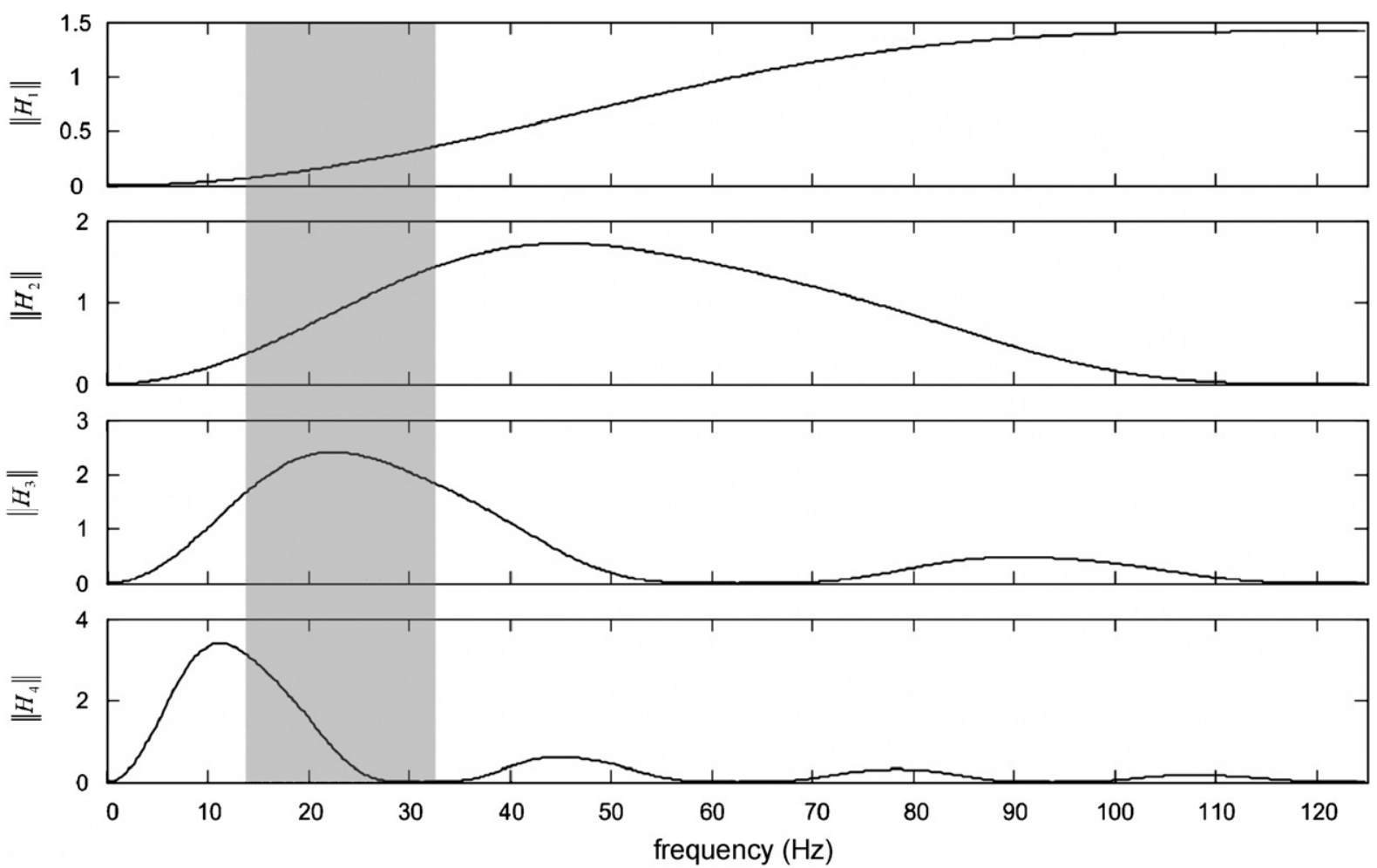

Fig. 1. The frequency response of the filter-bank of Coiflet-1. The gray section is the main frequency distribution of ECG artifacts.

pattern corresponding to the temporal features of the EEGs is the natural option. This reflects the fact that the shape of the detail at the $j$ th level, $D_{j}$, has considerable similarity with the wavelet basis via MRA processing. The original signal is convolved with wavelet filters in decomposition and reconstruction, as shown in Eqs. (2) and (3). This is to say that the wavelet coefficients contain periodic and shifted versions of the $j$ th level wavelet filter. The correlation coefficients are therefore estimated as a measure of similarity between the ECG artifacts and wavelet bases. Here $x$ and $y$ are two signals with $N$ samples:

$R_{(x, y)}=\frac{\sum_{i=1}^{N}\left(x_{i}-\bar{x}\right)\left(y_{i}-\bar{y}\right)}{\sqrt{\sum_{i=1}^{N}\left(x_{i}-\bar{x}\right)^{2} \sum_{i=1}^{N}\left(y_{i}-\bar{y}\right)^{2}}}$.

In addition, the properties of wavelet filter-banks must be examined [13]. The phases of the Symlet- 8 and Coiflet-1 filters at each decomposition level used in this work are approximately zero. When the phase function of a filter reaches zero phase or approximately zero phase, the time-shift function will be almost zero [13]. As a consequence, the result of decomposition has no time shift via MRA if the filters have zero phases. In contrast, time shift has already occurred at level 2 via the Daub- 2 filters. The time-shift functions of three selected wavelet filter-banks at each decomposition level can be found in [13].

The concepts of the basis and scale selection of WTs can be illustrated pictorially. Fig. 2 shows the procedures for detecting ECG artifacts and eliminating from an EEG. The suitable wavelet basis and decomposition scale are selected on the basis of frequency features, temporal morphology, and wavelet
EEGs with ECG Artifacts

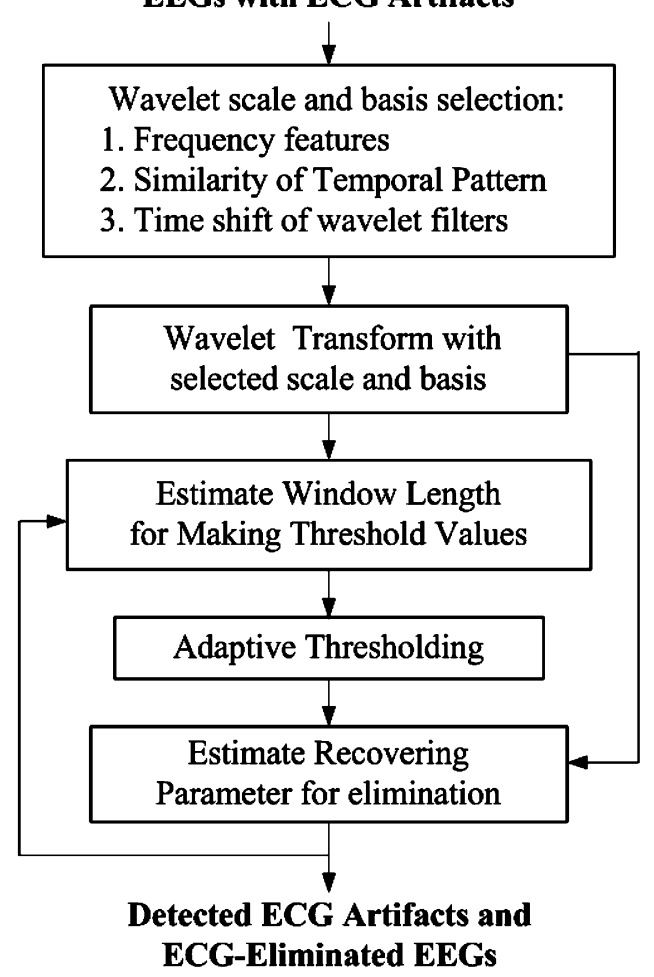

Fig. 2. The procedures for detecting ECG artifacts and eliminating from an EEG.

filter properties. The following sections utilize the foregoing concepts to perform artifact detection and elimination from EEGs. 


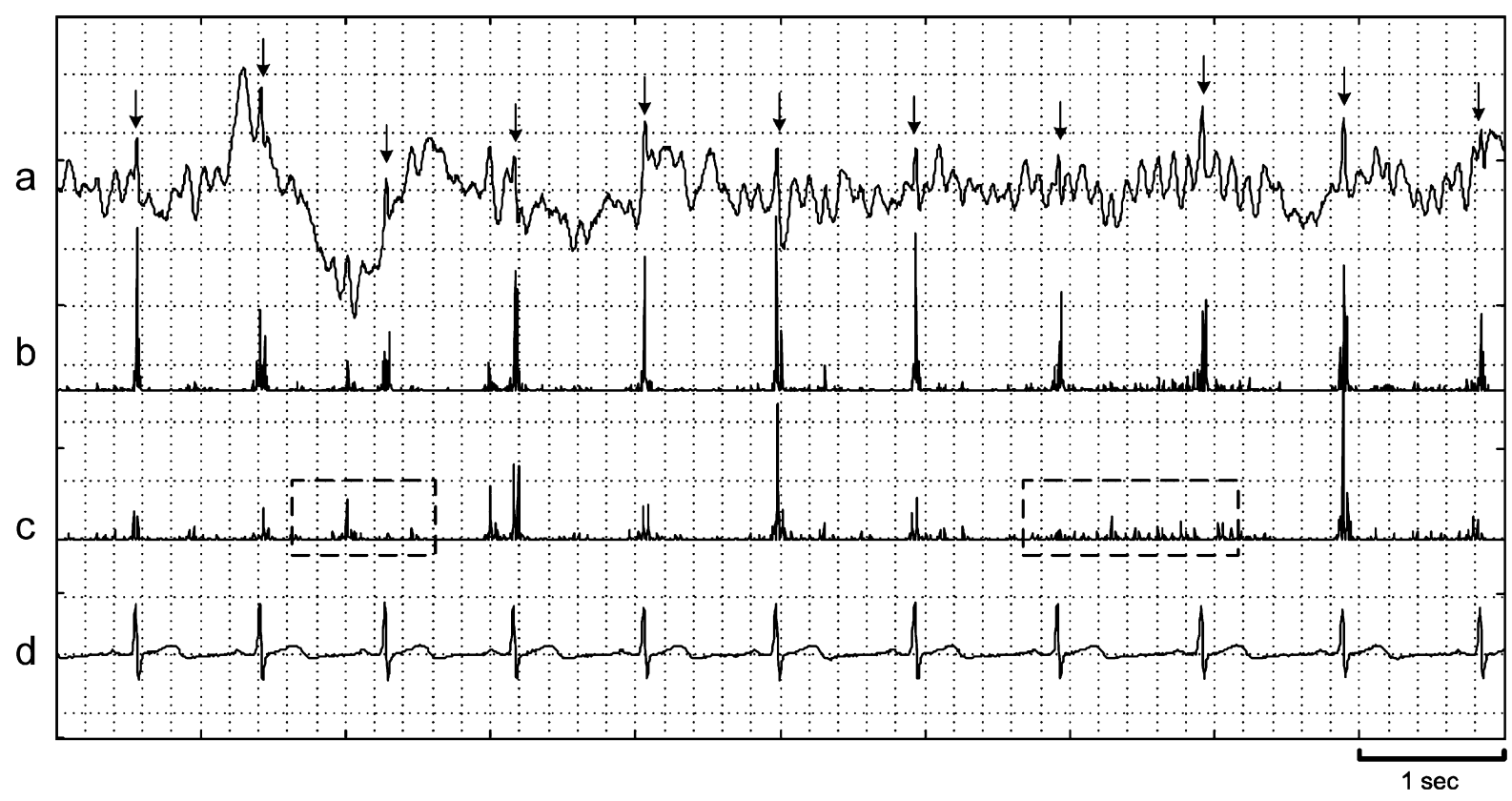

Fig. 3. Comparison of the energy coefficients at scales $2^{2}$ and $2^{3}$. (a) The raw EEG with ECG artifacts (indicated by arrows); (b) at scale $2^{2}$, the local maxima (peaks of energy coefficients) correspond to the R-peaks of ECG artifacts and the detections will be achieved correctly (indicated by arrows in (a)); (c) there are many false sections circled by dash line in the decomposition at $2^{3}$ scale, i.e., the local maxima do not correspond to the R-peaks of ECG artifacts (indicated by arrows in (a)); (d) the reference ECG channel for verifying the detection points.

\subsection{Wavelet-based ECG artifact detection and elimination}

The proposed method of detecting and eliminating ECG artifacts in EEGs is based on the foregoing concepts according to the power spectra of an ECG signal [14]. The proposed algorithm for the detection and elimination of ECG artifacts is described as follows.

\section{Step 1: Decomposition scale}

The gray part of Fig. 1 indicates the main frequency band of ECG artifacts, and it is clear that the frequency distribution of the QRS complex generally covers the scales of $2^{3}$ and $2^{2}$. Most artifact energy spectra are in the $2^{3}$ scale. Thus, we initially chose the scale $2^{3}$ to extract the R-peak of ECG artifacts, and this should be the general principle of decomposition. While inspection of the spectrum in Fig. 1 shows that the $2^{2}$ scale contains less energy than the $2^{3}$ scale, the extracted signal may avoid most variations like beta activity and sleep spindles at this scale. We therefore selected both $2^{3}$ and $2^{2}$ scales so that we could test the reliability of the algorithm via detection processing and simulation results. A comparison of results at different scales is shown in Fig. 3. A further discussion is presented in the next section.

\section{Step 2: Wavelet basis selection}

Based on the basis and scale selection criterion mentioned above, the first step of selection is to find a basis which has a sharp and spiky waveform consistent with spike-like ECG artifacts. We chose the basis, Coiflet-1, which has the greatest similarity with ECG artifacts, as shown in Figs. 4(a) and (b). Wavelet transformation using Coiflet-1 also avoids the problem of time shift due to the zero phase at each decomposition scale. Furthermore, Daub-2 (Fig. 4(d)) and Symlet-8 a

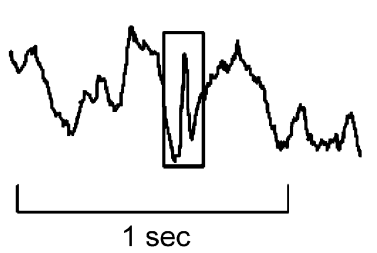

C

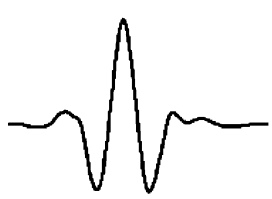

d

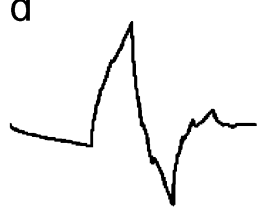

Fig. 4. (a) The ECG artifact in the EEG (in the frame); (b) Coiflet-1 wavelet basis; (c) Symlet-8 wavelet basis; (d) Daub-2 wavelet basis.

(Fig. 4(c)) — bases often used in the literature-were also used for the sake of comparison.

\section{Step 3: Adaptive thresholding}

The main goal of an adaptive thresholding algorithm is to reveal artifacts and make the focal points stand out. The threshold values depend on the local variation of the wavelet energy coefficients. The threshold values can be used to shrink the wavelet energy coefficients over specific intervals - called window length $L$ - to ensure correct detection. The wavelet details of the $j$ th decomposition level obtained via MRA are designated $D_{j}$, and the energy of coefficients at this resolution level is defined as $E_{j}(k)=D_{j}^{2}(k)$. An adaptive threshold scheme is 
a

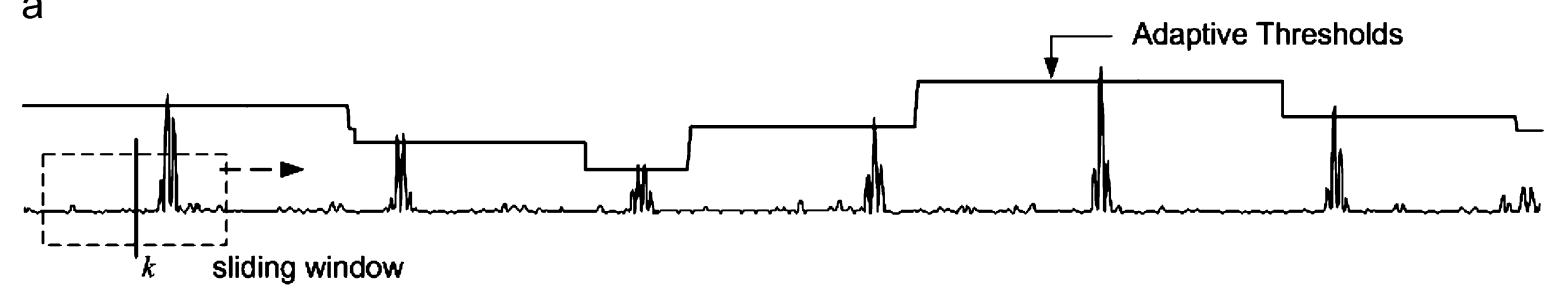

b

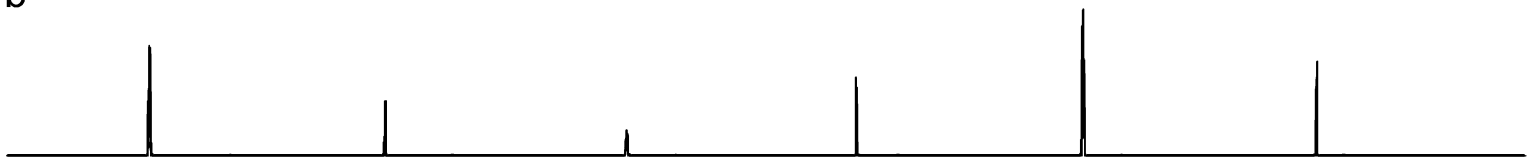

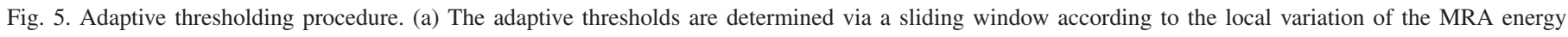
coefficients; (b) the detection points after adaptive thresholding.

proposed as follows:

$$
\begin{aligned}
& T(k)=\max \left[E_{j}\left(k-\frac{L}{2}: k+\frac{L}{2}\right)\right] \\
& \text { for } k=\frac{L}{2}, \frac{L}{2}+1, \ldots .
\end{aligned}
$$

Here $T(k)$ is the threshold at the $k_{\text {th }}$ time index and $L$ is the length of a moving window. In Fig. 5, the adaptive threshold values are determined according to the variations in each segment of energy coefficients, $E_{2}$, by a moving window. This procedure allows the determination of the local maximum of energy corresponding to the R-peak of ECG artifacts in a specific segment.

Furthermore, window length $L$ is also an important parameter affecting the correctness of the adaptive thresholding scheme. Detection will be achieved correctly when the window length is within the range of $I<L<2 I$, where $I$ is the interval length between two adjacent R-peaks ( $\mathrm{R}-\mathrm{R}$ intervals). In accordance with experimental results, the pre-defined length of a sliding window was set as 300 data points (1.2 s). Detection was achieved correctly as long as the R-R intervals were in the range of $0.6-1.2 \mathrm{~s}$. Nevertheless, $\mathrm{R}-\mathrm{R}$ intervals will change in long-term recordings due variation in heart rhythm. Window length was therefore updated every $10 \mathrm{~s}$ in order to ensure the correctness of detection. The current window length was determined on the basis of the mean value of the intervals between detection points during the previous $10 \mathrm{~s}$.

\section{Step 4: Elimination of ECG artifacts}

Suppose that an artifact-contaminated EEG can be expressed as $y(n)=x(n)+s(n)$, where $x(n)$ represents the original EEG without ECG artifacts and $s(n)$ represents the ECG artifacts. Using CWT, the extracted wavelet coefficients are taken as the estimated artifacts, $\hat{s}(n)$. Since we use the single scale wavelet decomposition, the WT cannot extract the total energy of the true artifacts, that is, the amplitude of extracted artifacts should be lower than that of the true ones. Therefore, the amplitude of the estimated artifacts $\hat{s}(n)$ must be multiplied by a constant $K$-called the recovering parameter- to approach the true amplitude of artifacts. Since the analysis of WT via Coiflet-1 has no time shift, the elimination of ECG artifacts can be achieved by directly subtracting the estimated artifacts $\hat{s}(n)$ from the contaminated EEGs $y(n)$. The artifact-cleaned EEGs $\hat{x}(n)$ can be defined as follows:

$\hat{x}(n)=y(n)-K \hat{s}(n)$.

Due to the variability of cardiac activity, the energy of ECG artifacts may change in long-term recordings for one subject or between subjects. The recovering parameter was estimated every $10 \mathrm{~s}$ for each recording in order to ensure the correctness of artifact elimination. The recovering parameter $K^{(i)}$ is estimated as the ratio between the amplitudes of EEGs and the extracted wavelet coefficients at the times of detection points (corresponding to the R-peak times of ECG artifacts) in the $i$ th 10 -s epoch. It can be expressed as $K^{(i)}=\sum_{j=1}^{m} y_{j}^{(i)}(p) / \sum_{j=1}^{m} \hat{s}_{j}^{(i)}(p)$, where $y_{j}^{(i)}$ and $\hat{s}_{j}^{(i)}$ are the respective relative amplitude of the $j$ th R-peak in $i$ th 10s epoch of an ECG-contaminated EEG and of the extracted wavelet details. The index $p$ is the time when the signal reaches the R-peak of ECG artifacts; it can be obtained from the time index of detection points. To prevent estimation error caused by failed detections, $K^{(i)}$ is regarded as a correct value within a reasonable range of $1-2.5 . K^{(i)}$ is substituted as the previous correct estimation, $K^{(i-1)}$, when the value is outside this range. Artifact-cleaned EEGs can be obtained by Eq. (7) using the estimated recovering values, $K^{(i)}$, in each 10 -s epoch.

\section{Evaluation and results}

The EEG recordings in this study were separated into two groups. The data in Group 1 consisted of "sleep" recordings obtained from the Polysomnographic Database established by Massachusetts Institute of Technology and Beth Israel Hospital (MIT/BIH) [15]. The EEGs in this group were originally contaminated with ECG artifacts. The performance of the proposed method based on CWT and DWT was derived for EEGs of different subjects and sleep stages.

The data in Group 2 consisted of "wakeful" recordings acquired from the Department of Neurology, National Taiwan University Hospital (NTUH). In order to verify the performance of the proposed method under various conditions, i.e., performance under different level of contamination and with correlation of signals before and after processing, the ECG artifacts 


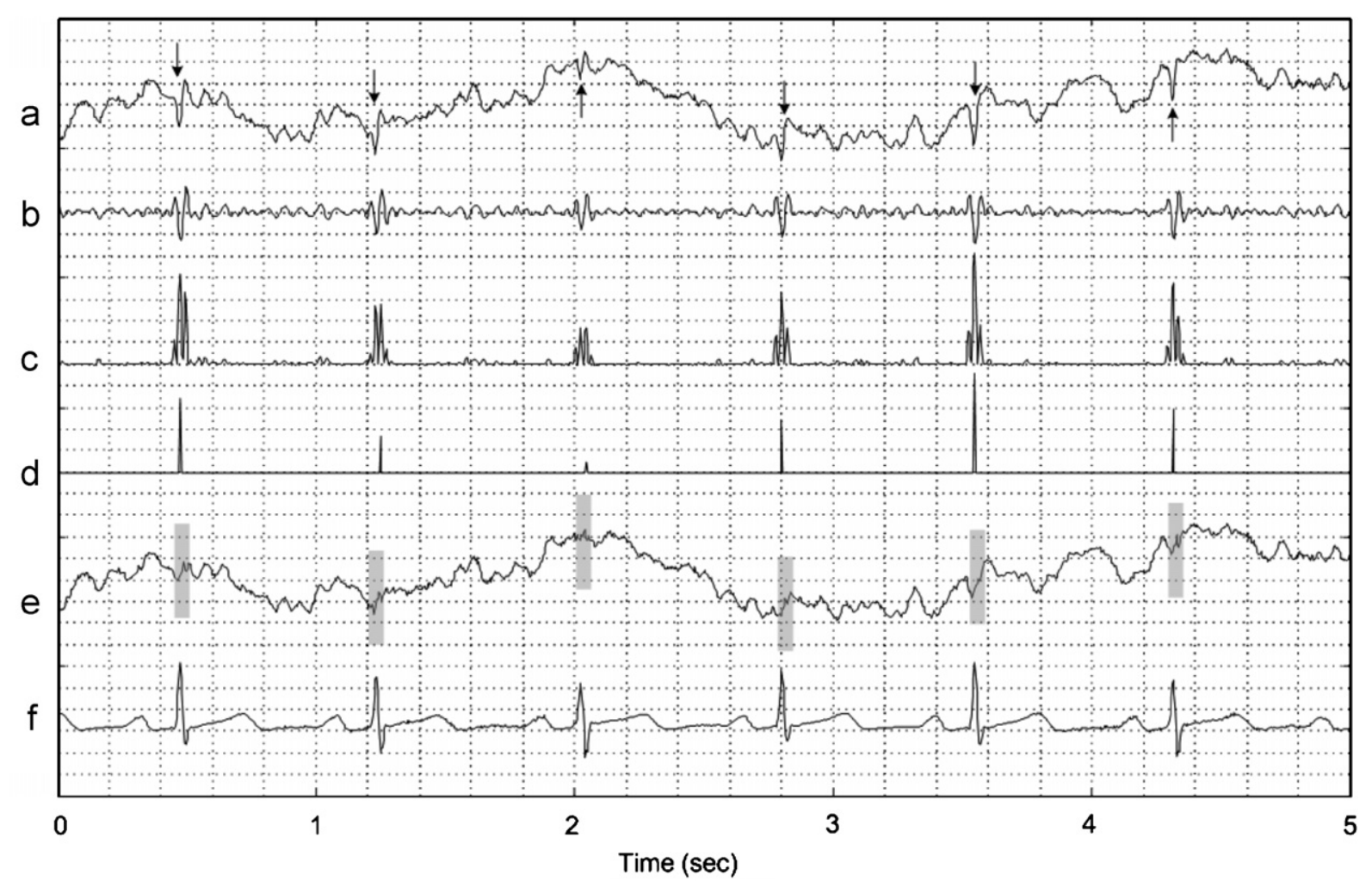

Fig. 6. The procedures of the automatic ECG artifact detection and elimination (sleep recordings): (a) the raw EEG contains ECG artifacts (arrows); (b) extracted ECG artifacts via CWT with a Coiflet-1 basis; (c) the wavelet energy coefficients (powers of extracted ECG artifacts); (d) the detection of ECG R-peaks after using the adaptive thresholding procedure; (e) the artifact-cleaned EEG; (f) the synchronous ECG signal was used to verify the detection results.

in this group were taken from a synchronous ECG channel and incorporated into the EEG signals by linear addition at various amplitudes.

\subsection{Performance indices for statistical assessment}

The rules for scoring the results of automatic detection addressed the two main considerations of the identification of detections and the time accuracy of detections. Detection was taken to be completed correctly when these two criteria were both satisfied. The first rule is to count the false positive (FP) and false negative (FN) detections, which are described as follows:

$F N$ beats: the number of missed detection while ECG artifacts are present.

FP beats: the number of additional unexpected detections when the ECG artifacts are not present.

The second rule is to identify the time accuracy of detections. This rule specifies that a detection is performed only when it is located within a range of $0.1 \mathrm{~s}$ around the true temporal location of an ECG artifact. The two scoring rules were used to test the robustness and accuracy of the proposed algorithm.

Spike-to-EEG energy ratio (SER): Elimination performance was evaluated by estimating the SER [8] before and after the elimination procedures. The SER is defined as the ratio of the mean energy of a spike region (QRS wave) to the mean energy of the background EEG in a recording:

$\mathrm{SER}=\frac{\left(1 / N_{k}\right) \sum_{k=1}^{N_{k}}\left(\left(1 / N_{S}^{(k)}\right) \sum_{n=1}^{N_{S}^{(k)}} \mathrm{SE}^{(k)}(n)\right)}{\left(1 / N_{B}\right) \sum_{n=1}^{N_{B}} \operatorname{EE}(n)}$.

Here $\mathrm{SE}^{(k)}(n)$ represents the signal energy of the $k_{\mathrm{th}}$ QRS region with $N_{S}^{(k)}$ samples; $N_{k}$ is the number of total spike segments in the recordings; $\operatorname{EE}(n)$ represents the signal energy of the EEG background in the non-spike region with $N_{B}$ samples in the recordings. The estimated SER can be regarded as the degree of ECG artifact contamination in both groups and can be used to regulate the added ECG energy level in the cases in Group 2.

Correlation: The correlation coefficient is a normalized measure of the degree of linear correlation between two signals. It is used to verify the correlation between signals before and after processing in the NTUH cases. Correlation coefficients are defined in Eq. (5), where $x$ and $y$ are signals with $N$ samples, and $R(x, y)$ is the correlation coefficient between $x$ and $y$. The correlation coefficients used in this work are described as follows:

$R_{(\mathrm{rEEG}, \mathrm{rEEG}+\mathrm{rECG})}$ : The mean correlation between the original non-ECG-contaminated EEG (rEEG, Fig. 7(a)) and the ECG-contaminated EEG (rEEG + rECG, Fig. 7(c)) in the recordings.

$R_{(\mathrm{rECG}, \mathrm{eECG})}$ : The mean correlation between original added ECG spikes (rECG, Fig. 7(b)) and the ECG spikes estimated by WT (eECG, Fig. 7(g)) in the recordings. 
$R_{(\mathrm{rEEG}, \mathrm{eEEG})}$ : The mean correlation between original nonECG-contaminated EEG (rEEG, Fig. 7(a)) and the artifactcleaned EEG (eEEG, Fig. 7(g)) in the recordings.

\subsection{Group 1: sleep recordings}

\subsubsection{Subjects and recordings}

The selected EEG records from the MIT/BIH database were originally contaminated with ECG artifacts. The three subjects were male, aged 33, 38, and 44, with weights of 95 , 145 and $89 \mathrm{~kg}$, respectively, and suffering from obstructive sleep apnea syndrome. EEG data were acquired with electrodes placed at C4-A1, O2-A1, and C3-O1 in accordance with the standard 10-20 electrode placement system. The sampling rate was $250 \mathrm{~Hz}$ and the resolution of the EEG data was 12bit. Supplemental EMG and EOG records were also included to determine sleep stages manually by RK rules [16]. The recordings of the three subjects were employed to evaluate the overall performance of the proposed algorithm for different subjects, sleep stages, and WT types (CWT and DWT). In this study, recordings containing ECG artifacts were arbitrary acquired; each test recording was about $60-90 \mathrm{~min}$ in length. A simultaneous ECG channel was used in all cases as a reference when we scored detection results. Five sleep stages and wakeful stages were also included in the selected recordings.

\subsubsection{Experimental results}

Fig. 6 shows adaptive thresholding procedure for automatic ECG artifact detection and elimination procedures. The raw EEG still contains ECG artifacts (arrows) in Fig. 6(a). The ECG artifacts were extracted via CWT with a Coiflet-1 basis, as shown in Fig. 6(b). The wavelet energy coefficients (powers of extracted ECG artifacts) are shown in Fig. 6(c). As shown in Fig. 6(d), the detection of ECG R-peaks is achieved after using the adaptive thresholding procedure (as shown in Fig. 5). The artifact-cleaned EEG is shown in Fig. 6(e). The synchronous ECG signal was used to verify the detection results, as shown in Fig. 6(f).

\subsubsection{Statistical assessment results}

The number of heart beats was 16,690 over a total period of $231 \mathrm{~min}$, with a mean heart rate of 72.25 beats $/ \mathrm{min}$. The recordings were utilized to test performance of the proposed algorithm via CWT and DWT, respectively. The performance evaluation for each recording is listed in Table 1 . The proposed algorithm via DWT yielded an overall 1.61\% FN detection rate and an overall $0.61 \%$ FP detection rate. Via CWT, the algorithm's FN detection and FP detection rates were 1.59\% and $0.53 \%$, respectively. The original mean SER (SERr), 5.64, was reduced to 1.41 (the artifact-cleaned SER, SERe). The detection performance for individual sleep stages is shown in Table 2.

In Table 2, the failed detection rates in stages 3 and 4 were less than $1.06 \%$ via either CWT or DWT. The highest failed detection rates occurred during sleep stage 1 and the wakeful stage and were roughly $3.20-5.49 \%$. The failed detection rate during rapid eye movement (REM) stage was a little lower than those during the wakeful stage and stage 1, which were $3.24 \%$ via DWT and $2.50 \%$ via CWT. The failed detection rates during stage 2 were $1.65 \%$ and $2.03 \%$ via DWT and CWT, respectively.

\subsection{Group 2: wakeful recordings}

\subsubsection{Subjects and recordings}

The recordings of 10 normal wakeful subjects from NTUH were randomly selected from data previously recorded with an EEG 1000 system (Nihon Kohden Co., Japan) with a sampling rate of $200 \mathrm{~Hz}$. Two subjects were male and eight were female, aged from 11 to 64 (mean age of 28.5). The recordings included the data from six EEG channels (FP2-A2, F3-A1, C4-A2, T5$\mathrm{A} 1, \mathrm{P} 4-\mathrm{A} 2$, and $\mathrm{O} 1-\mathrm{A} 1$, according to the standard 10-20 electrode placement system). Each recording was about $4-5 \mathrm{~min}$ in length.

Since the locations of the EEG channels were chosen to cover various locations on the scalp, the wakeful EEG with more noise could be used to test the robustness of the proposed method. The reference ECG channel was filtered in the range of $5-40 \mathrm{~Hz}$ to remove most non-spike waves and then added to each EEG channel with various amplitude levels to verify the detection and elimination performance. A general QRS detection algorithm [17] was used to detect QRS peaks of the reference ECG channel used to evaluate performance.

\subsubsection{Experimental results}

Fig. 7 shows step by step automatic ECG artifact detection and elimination procedures. Fig. 7(a) shows the raw EEGs (rEEGs) without ECG artifacts. In Fig. 7(c), ECG-contaminated EEGs (rEEGs + rECGs) are constructed by adding filtered ECGs to rEEGs, as shown in Fig. 7(b). In this example, the ECG SER was set as 10 (SER will be described in the next section). The EEGs with artifacts are then transformed into level 2 detail, $D_{2}$, via WT with a Coiflet-1 basis, as shown in Fig. 7(d). The extracted detail (estimated ECGs and eECGs) reveals the hidden artifacts. In Fig. 7(e), the wavelet energy coefficients are computed and the extracted artifacts are even more evident than at the previous stage. Finally, as shown in Figs. 7(f) and (g), the detected temporal points and the energies of ECG R-peaks are determined following the adaptive thresholding procedure, and the artifact-cleaned EEGs (eEEGs) are also obtained. To see the nature of the residual artifacts, Fig. 7(h) shows the residual signal: raw EEG compares to ECGcleaned EEG (rEEG-eEEG).

\subsubsection{Statistical assessment results}

The total number of ECG artifacts from six EEG channels at five SER levels was 102,120. The length of recordings was $42.97 \mathrm{~min}$, with a mean heart rate of 79.22 beats $/ \mathrm{min}$. The detection and elimination performance for each recording at specific SER levels is listed in Table 3. The mean failed detection (FD, equal to FN + FP) rate of all recordings at all SER 
Table 1

Performance evaluation with respect to subjects (MIT/BIH cases) via DWT and CWT

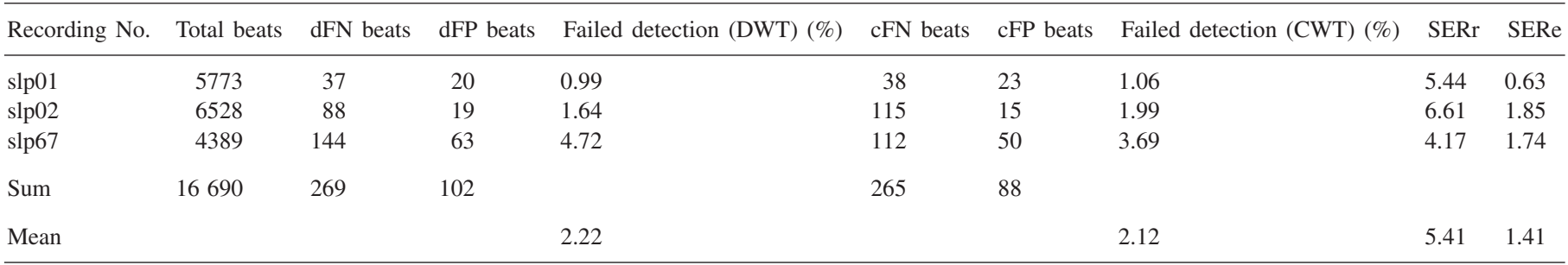

dFN/dFP beats: the number of false negative/ positive detections after DWT; cFN/cFP beats: the number of false negative/ positive detections after CWT; SERr: original SER; SERe: ECG-eliminated SER.

Table 2

Performance evaluation with respect to different sleep stages (MIT/BIH cases) via DWT and CWT

\begin{tabular}{lllllll}
\hline & Stage 1 & Stage 2 & Stage 3 & Stage 4 & REM & Wakeful \\
\hline Total beats & 455 & 7619 & 940 & 2124 & 3118 \\
dFP beats & 17 & 101 & 5 & 8 & 2434 \\
dFN beats & 8 & 25 & 4 & 4 & 60 \\
Failed detection (DWT) (\%) & 5.49 & 1.65 & 0.96 & 0.56 & 38 \\
cFP beats & 17 & 127 & 6 & 5 & 3.24 \\
cFN beats & 7 & 28 & 4 & 3 & 61 \\
Failed detection (CWT) (\%) & 5.27 & 2.03 & 1.06 & 0.38 & 17 \\
\hline
\end{tabular}

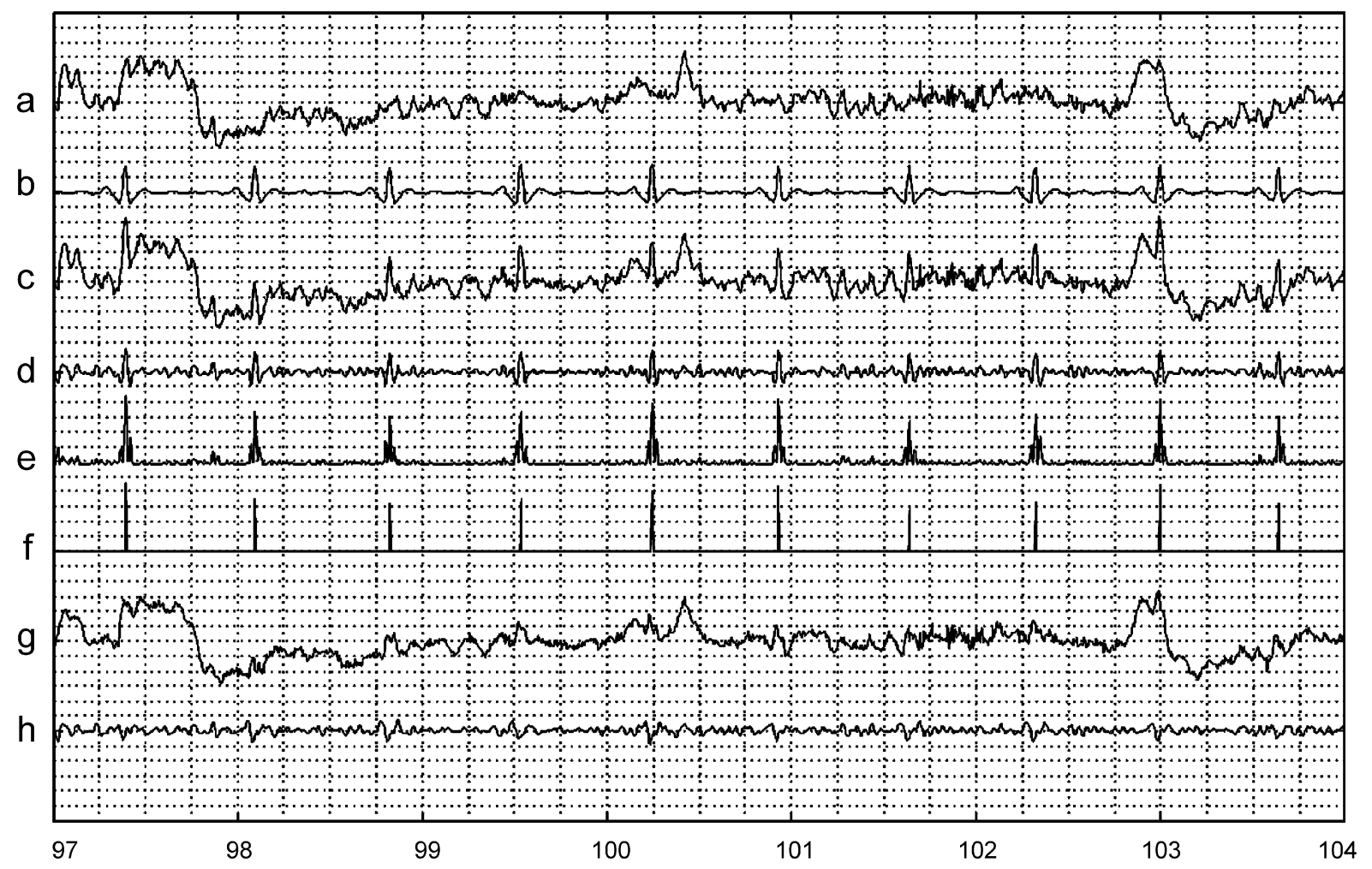

Fig. 7. The procedures of the automatic ECG artifact detection and elimination (wakeful recordings): (a) the raw EEG (rEEG) without ECG artifacts; (b) the original ECG channel filtered in the range of $5-40 \mathrm{~Hz}(\mathrm{rECG})$; (c) the ECG-contaminated EEG (rEEG + rECG); (d) the extracted ECG (eECG) from the ECG-contaminated EEG by Coiflet-1; (e) the energy of extracted ECG; (f) the detection after adaptive thresholding on (e); (g) the ECG-cleaned EEG (eEEG) after elimination procedure; (h) the residual signal: raw EEG compares to ECG-cleaned EEG (rEEG-eEEG).

level was $2.09 \%$. The mean and standard deviation of FD for all subjects at various electrode locations are summarized in Table 4.
The correlations between signals before and after processing at distinct SER levels are listed in Table 5. As for ECG spikes extraction, the mean correlations between rECG and 
Table 3

Performance evaluation with respect to subjects (NTUH cases) at distinct SER level

\begin{tabular}{|c|c|c|c|c|c|c|c|c|c|c|}
\hline \multirow[t]{3}{*}{ Recording No. } & \multicolumn{10}{|l|}{ SERr } \\
\hline & \multicolumn{2}{|l|}{3} & \multicolumn{2}{|l|}{5} & \multicolumn{2}{|l|}{10} & \multicolumn{2}{|l|}{15} & \multicolumn{2}{|l|}{20} \\
\hline & FD (\%) & SERe & $\overline{\mathrm{FD}(\%)}$ & SERe & $\overline{\mathrm{FD}(\%)}$ & SERe & $\overline{\mathrm{FD}(\%)}$ & SERe & $\overline{\mathrm{FD}(\%)}$ & SERe \\
\hline ntuh01 & 11.75 & 1.39 & 2.66 & 2.10 & 0.41 & 3.44 & 0.14 & 4.74 & 0.00 & 6.03 \\
\hline ntuh02 & 8.60 & 0.85 & 2.92 & 1.24 & 0.43 & 2.01 & 0.16 & 2.79 & 0.16 & 3.57 \\
\hline ntuh03 & 3.61 & 1.34 & 1.91 & 2.04 & 0.67 & 3.40 & 0.36 & 4.75 & 0.26 & 6.09 \\
\hline ntuh04 & 2.43 & 1.23 & 0.63 & 1.87 & 0.09 & 3.11 & 0.18 & 4.35 & 0.18 & 5.58 \\
\hline ntuh05 & 1.89 & 1.28 & 0.57 & 1.97 & 0.28 & 3.32 & 0.28 & 4.65 & 0.28 & 5.97 \\
\hline ntuh06 & 2.93 & 1.40 & 0.21 & 2.07 & 0.10 & 3.29 & 0.00 & 4.47 & 0.00 & 5.64 \\
\hline ntuh07 & 5.92 & 1.58 & 2.99 & 1.43 & 1.03 & 2.40 & 0.57 & 3.42 & 0.57 & 4.45 \\
\hline ntuh08 & 12.43 & 1.56 & 5.41 & 1.76 & 0.39 & 3.11 & 0.24 & 4.49 & 0.15 & 5.89 \\
\hline ntuh09 & 8.36 & 1.36 & 1.91 & 1.60 & 0.05 & 2.80 & 0.00 & 4.02 & 0.00 & 5.25 \\
\hline ntuh10 & 13.46 & 0.79 & 5.57 & 0.83 & 1.14 & 1.38 & 0.34 & 1.95 & 0.27 & 2.53 \\
\hline Mean $\mathrm{FD} \pm \mathrm{SD}$ & $7.14 \pm 4.17$ & $1.28 \pm 0.27$ & $2.48 \pm 1.87$ & $1.69 \pm 0.42$ & $0.46 \pm 0.38$ & $2.83 \pm 0.69$ & $0.23 \pm 0.17$ & $3.96 \pm 0.95$ & $0.19 \pm 0.17$ & $5.10 \pm 1.2$ \\
\hline
\end{tabular}

FD: failed detection; SD: standard deviation of FD among subjects.

Table 4

Performance evaluation with respect to electrode location (NTUH cases)

\begin{tabular}{llc}
\hline Electrode location & Mean FD $(\%)$ & SD $(\%)$ \\
\hline FP2-A2 & 2.43 & 2.64 \\
F3-A1 & 1.92 & 1.33 \\
C4-A2 & 1.49 & 1.42 \\
T5-A1 & 1.66 & 1.84 \\
P4-A2 & 1.59 & 1.44 \\
O1-A1 & 3.29 & 2.39 \\
\hline
\end{tabular}

SD: standard deviation of FD rate among subjects at the location.

Table 5

Correlations between signals at distinct SER levels (NTUH cases)

\begin{tabular}{lcclll}
\hline Correlation & \multicolumn{2}{l}{ SERr } & & & \\
\cline { 2 - 6 } & 3 & 5 & 10 & 15 & 20 \\
\hline$R_{\text {(rEEG,rEEG+ECG) }}(\%)$ & 85.15 & 76.57 & 70.26 & 65.35 & 61.38 \\
$R_{\text {(rECG,eECG) }}(\%)$ & 80.32 & 82.45 & 83.24 & 83.66 & 83.93 \\
$R_{\text {(rEEG,eEEG) }}(\%)$ & 85.01 & 79.48 & 75.00 & 72.69 & 69.43 \\
\hline
\end{tabular}

$R_{\text {(rEEG,rEEG+ECG) }}$ : mean correlation between rEEG and rEEG + rECG

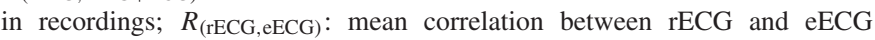
in spike regions; $R_{(\mathrm{rEEG}, \mathrm{eEEG})}$ : mean correlation between rEEG and eEEG in recordings.

eECG in spike regions, $R_{(\mathrm{rECG}, \mathrm{eECG})}$, is above $80 \%$ at all SER levels. $R_{\text {(rEEG,rEEG }+\mathrm{rECG})}$ and $R_{\text {(rEEG,eEEG) }}$ reveal correlations between EEGs before and after elimination procedure.

\section{Discussion}

This study proposes the concepts of wavelet basis/scale selection and wavelet-based methodology for ECG artifact detection and elimination. The proposed method achieves pretty good performance. The key element of the proposed methodology is the selection of suitable wavelet basis. The selected basis can improve the overall performance of detection and elimination directly from EEG without an additional ECG signal.
The wavelet bases used with our algorithm avoid the problem of time shift after processing. This advantage enhances temporal accuracy when using the WT technique. A wavelet basis with pattern corresponding to EEG/ECG characteristics allows a good projection from the characteristics via WT, and the extracted signal can match the specific waveform even more closely.

It should be noted that $2^{2}$ was chosen as the alternative decomposition scale. Although the energy at the $2^{2}$ scale is lower than that of the $2^{3}$ scale (see Fig. 1), extraction results at the $2^{2}$ scale are better than that at the $2^{3}$ scale, as shown in Fig. 4. This is because the most beta activity and sleep spindles are also extracted simultaneously at the $2^{3}$ scale. In other words, the extracted wavelet coefficients at the $2^{3}$ scale contain much unnecessary EEG activity. It is clear that results at the $2^{2}$ scale are much better, and the relative high energy coefficients correspond to the R-peaks. In addition, results at the $2^{3}$ scale have much false extraction, as shown in Fig. 4(c).

Since the sampling frequency $\left(f_{\mathrm{s}}\right)$ of EEG might be different in other applications, it should be noted that the wavelet scale used to extract artifacts will not be fixed. The selection of a proper scale is relative with respect to the sampling frequency of the EEG signal. As mentioned above, the most proper scale for ECG artifact detection and elimination will be $2^{(n-1)}$. The decomposition level $n$ can be determined by a manner that the main frequency band of the wavelet filter at $2^{n}$ scale can locate in the range of $10-30 \mathrm{~Hz}$ approximately (main frequency band at each level can be defined for different sampling frequency, see Section 2.1). For sampling frequency at $200 \mathrm{~Hz}$ in our case, the main frequency band of the wavelet filter at scale $2^{3}$ is in the range of $12.5-25 \mathrm{~Hz}$, so we choose $2^{3-1}$ as the decomposition scale.

The detections at each stage showed high adaptability and reliability in the diverse environment of the background EEG, as shown in Table 3. It is clear that a suitable basis and decomposition level make the detection algorithm much more powerful and avoid the effects of EEG variability, even when the artifacts are not visually obvious. Due to the transformation 
without time shift, the extracted ECG artifacts can be directly subtracted from the contaminated EEG. It should be noted that the phase of ECG artifacts may be different in recordings (positive or opposite). However, the types of phase do not influence the results of elimination. The phase of extracted signals depends on that of the ECG artifacts. Spikes were also removed by the same elimination procedure.

\subsection{MIT/BIH database}

The application of the proposed algorithm to subjects with obstructive sleep apnea syndrome showed excellent results. The manual scoring statistics and assessment in Table 1 indicate that detection performance significantly depends on the subject, which means that the properties of subjects' EEG signals influence the detection rate. EEG activities and artifacts are likely to remain consistent for one subject, but may differ considerably between subjects. For instance, the EEG of subject slp01 displayed less contaminating high frequency noise than the other recordings, and detection performance for subject slp01 was therefore superior to that for the other subjects. On the other hand, the EEG of subject slp67 was frequently contaminated with much more high amplitude EMG noise. When this situation occurs, the extracted ECG signal is mixed with EMG noise and failed detections tend to occur relative to the quantity of noise. It should be noted that detection can also be achieved accurately when EMG noise is not severe (relative amplitude is not much greater than background EEG).

In Table 2, the failed detection rates at stages 3 and 4 were very low (less than $1 \%$ ) regardless of whether DWT or CWT was used. This suggests that the background frequency of the EEGs was lower than ECG artifacts during deep sleep, and also indicates that the detection algorithm worked well. The highest failed detection rates occurred during the wakeful stage, sleep stage 1, and REM stage. This is explained by the fact that the many EMG artifacts and body movements occurring during these stages contaminated the EEGs. ECG spikes and the frequency bands of these disturbances overlapped each other. The failed detection rate rose with an increased number of disturbances. It should be noted that the total beats in recordings during stage 1 were much less than during other stages (the original recordings contained many fewer episodes of stage 1 sleep). The failed detection rate during stage 1 in our experiments was thus higher than during the wakeful state. Manual scoring experience suggests that detection during the wakeful stage (when most EMG artifacts and body movements occur) is quite difficult. The occurrence of sleep spindles during stage 2 led to a higher detection rate during stage 2 higher than during stages 3 and 4. Another reason for failed detections was the occurrence of unexpected spikes, which had a frequency very close to that of ECG artifacts. The above-mentioned situations were not disregarded in the final manual score, which means that all detections were counted even though the ECG artifacts were almost invisible. The only situations in which scores were not taken into account were sleep stages that were labeled as MT (moving time), when the EEG amplitude was more than
$300 \mu \mathrm{V}$ (typical threshold of normal EEGs), and when the EEGs had no contaminating ECG artifacts.

Tables 1 and 3 show comparative results for DWT and CWT. While CWT should generally yield more accurate analytical results than DWT due to its continuous scales and shifts, our experiments indicated that the performance of CWT is not entirely superior to that of DWT. We found that CWT produced more false detections than DWT in some cases. The reason for this result was that the use of DWT with the proposed algorithm also employed the $2^{2}$ scale, and the filters shifted with a four sampling interval, i.e., $\Delta t=4 / 250=0.016 \mathrm{~s}$. This short shift interval was sufficient to achieve the desired accuracy for detection of ECG artifacts in the experiments. DWT is thus more efficient with fast algorithms and demonstrated satisfactory performance in the experiments.

\subsection{NTUH clinical recordings}

The proposed method was validated again using the recordings with supplemental experimental indices. Data for all subjects were recorded while they were in a wakeful condition. As mentioned above, detections were difficult to achieve during the wakeful stage since most noise and EEG/ECG variation generally occurred during this stage. The wakeful stage recordings can therefore be considered as relatively extreme cases. Looking at real ECG-contaminated cases, the setting of SER levels should be sufficient to cover most situations in which ECG artifacts occur.

In the very low ECG-contaminated cases (e.g., $\mathrm{SER}<3$ ), ECG artifacts were almost invisible and tended to be submerged in the background EEG. The failed detection rate thus increased with the reduction of SER. The reason for this was that in some recordings, especially at low SER levels, ECG spikes were not sharp enough to be differentiated from high frequency EEG activity, implying that the failed detection rate increases when ECG artifacts are unusually submerged in the EEG signals. However, the overall detection performance did not deteriorate severely and still remained acceptable under such conditions. As for the aspect of elimination performance, according to the standard deviations of SERes, the differences between artifact-cleaned SERs (SERes) and other recordings with the same SERrs were not significant. The waveforms and amplitudes of extracted ECG spikes were therefore adapted to those of real ECG spikes. The proportion subtracted from the EEG in the spike regions was thus equivalent for each pair (extracted and real) of spikes. Furthermore, ECG artifacts were almost completely eliminated in most real ECG-contaminated situations (SERr $\square$ 10) according to the satisfactory results of SERe $($ SERe $<3)$.

In terms of the detection performance for specific electrode locations, we can see that failed detections occurred most frequently in the frontal (FP2-A2) and occipital (O1-A1) positions. However, according to the standard deviations in Table 4, the FD rate for identical locations was quite different in the case of different subjects. The index $R_{\mathrm{CC}}$ was above $80 \%$ for all SER levels and it could be interpreted that the correlation between 
contaminated and extracted ECGs in spike regions after transformation is high. Further, $R_{(\mathrm{rEEG}, \mathrm{rEEG}+\mathrm{rECG})}$ and $R_{(\mathrm{rEEG}, \mathrm{eEEG})}$ described the improvement of correlations of contaminated and eliminated EEGs with the original non-contaminated EEGs. Improvements suggest that the removal of ECG artifacts causes the waveform of eEEGs to more closely approximate those of the rEEGs. The background EEGs in non-spike regions also maintained their original waveforms and were not disturbed by the elimination procedure. At a SER level equal to 3, the $R_{(\mathrm{rEEG}, \mathrm{eEEG})}$ was slightly lower than the $R_{(\mathrm{rEEG}, \mathrm{rEEG}+\mathrm{rECG})}$. Since the effects of spikes are relatively less than those of nonspike regions in extracted ECG at the low SER levels, the subtraction of extracted ECGs from the EEGs influenced the promotion of $R_{\text {(rEEG,eEEG) }}$. It should be noted that, in practice, it was not necessary to perform elimination procedures since the ECG artifacts were almost submerged in other EEG activities.

Unlike previous studies conducted under restricted limited conditions [8], under unrestricted conditions the average FD rate of single channel cases (MIT/BIH) was $2.12 \%$ and that of multi-channel cases (NTUH) was $2.10 \%$. The SER of artifactcleaned EEGs was within the acceptable contamination limit for clinical practice. The proposed algorithm's conceptual clarity, simple procedures, and high efficiency make it very suitable for other applications.

\section{Conclusions}

A reliable and feasible wavelet-based methodology for ECG artifact detection and elimination is presented in this study. The main contribution of the proposed method is the successful application of the concepts of wavelet basis and scale selection to biomedical signals. The proposed algorithm provides a pretty good performance in ECG artifact detection and elimination. The reliability and validation of the proposed method were verified by various experimental indices. The experimental performance of the method shows that WT is an excellent analytical method when the optimal scale and a suitable basis are employed.

The specific frequency band and temporal features of other waveforms, such as epilepsy spikes, sleep spindles, and Kcomplexes, have rhythm extraction and ECG artifact detection aspects similar to those addressed by our algorithm. We believe that our proposed method can also be used for other applications involving these types of waveforms.

\section{Summary}

An automatic analysis method for detecting and eliminating electrocardiograph (ECG) artifacts in electroencephalography (EEG) is proposed in this paper. Considering the properties of wavelet filters and the relationship between wavelet basis and EEG characteristics, the criterion for selecting the suitable wavelet bases and scales used in EEG analysis is developed. Based on the selection criterion of wavelet basis, the EEG analysis via the selected bases is without suffering time shift in the process of decomposition and detection/elimination after wavelet transformation. The effective separation of alpha, beta rhythms and slow wave activity is achieved and the results conform to the clinical concern. Based on the similar concepts of proposed wavelet analysis, an automatic and adaptive method with high reliability to detect and eliminate ECG artifacts from EEGs without an additional synchronous ECG channel is also developed. An extensive of performance evaluations with respect to both of MIT/BIH database and NTUH clinical subjects is conducted. The achieved average detection rate of ECG artifacts is above $97.5 \%$. After elimination procedure, the interference of ECG artifact is almost removed below an acceptable contamination level for clinical practice. The performance evaluations have demonstrated that the proposed wavelet-based approach can be successfully implemented for biomedical signal processing with accurate analysis.

\section{References}

[1] R. Agarwal, J. Gotman, Computer-assisted sleep staging, IEEE Trans. Biomed. Eng. 48 (12) (2001) 1412-1423.

[2] H.S. Liu, T. Zhang, F.S. Yang, A multistage, multimethod approach for automatic detection and classification of epileptiform EEG, IEEE Trans. Biomed. Eng. 49 (12) (2002) 1557-1566.

[3] T. Kalayci, O. Ozdamar, Wavelet preprocessing for automated neural network detection of EEG spikes, IEEE Eng. Med. Biol. Mag. 14 (2) (1995) $160-166$.

[4] S. Flavio, E. Mario, Automatic detection of epileptiform activity by single-level wavelet analysis, Clin. Neurophys. 110 (2) (1999) 239-249.

[5] M. Cabrero-Canosa, E. Hernandez-Pereira, V. Moret-Bonillo, Intelligent diagnosis of sleep apnea syndrome, IEEE Eng. Med. Biol. Mag. 23 (2) (2004) 72-81.

[6] A.A. Petrosiana, D.V. Prokhorovb, W. Lajara-Nansona, R.B. Schiffera, Recurrent neural network-based approach for early recognition of Alzheimer's disease in EEG, Clin. Neurophys. 112 (8) (2001) $1378-1387$.

[7] M. Nakamura, H. Shibasaki, Elimination of EKG artifacts from EEG records: a new method of noncephalic referential EEG recording Electroencephalogr, Clin. Neurophys. 66 (1987) 89-92.

[8] H.J. Park, D.U. Jeong, K.S. Park, Automated detection and elimination of periodic ECG artifacts in EEG using the energy interval histogram method, IEEE Trans. Biomed. Eng. 49 (12) (2002) 1526-1533.

[9] M. Unser, A. Aldroubi, A review of wavelets in biomedical applications, Proc. IEEE 84 (4) (1996) 626-638.

[10] A. Hojjat, Z. Ziqin, D. Nahid, Analysis of EEG records in an epileptic patient using wavelet transform, J. Neurosci. Methods 123 (1) (2003) 69-87.

[11] M. Shen, L. Sun, F.H.Y. Chan, Method for extracting time-varying rhythms of electroencephalography via wavelet pocket analysis, IEE Proc. Sci. Meas. Technol. 148 (1) (2001) 23-27.

[12] S. Mallat, A Wavelet Tour of Signal Processing, Academic Press, San Diego, 1999 pp. 255-258.

[13] D.B. Percival, A.T. Walden, Wavelet Methods for Time Series Analysis, vol. 154, Cambridge University Press, New York, 2000 pp. 115-121.

[14] N.V. Thakor, J.G. Webster, W.J. Tompkins, Estimation of QRS complex power spectra for design of a QRS filter, IEEE Trans. Biomed. Eng. 31 (1984) $702-705$.

[15] Y. Ichimaru, G.B. Moody, Development of the polysomnographic database on CD-ROM, Psychiatry Clin. Neurosci. 53 (2) (1999) $175-177$.

[16] A. Rechtschaffen, A. Kales (Eds.), A manual of standardized terminology, in: Techniques and Scoring System for Sleep Stage of Human Subjects, BIS/BRI, UCLA, Los Angeles, CA, 1968.

[17] J. Pan, W.J. Tompkins, A real-time QRS detection algorithm, IEEE Trans. Biomed. Eng. 32 (3) (1985) 230-236.

Joe-Air Jiang was born in Taipei, Taiwan, in 1963. He received the M.S. and Ph.D. degrees both in electrical engineering from National Taiwan University, Taipei, Taiwan, in 1990 and 1999, respectively. From 1990 to 2001, 
he was with Kuang-Wu Institute of Technology, Taipei, Taiwan. Currently, he is an Associate Professor in the Department of Bio-Industrial Mechatronics Engineering at National Taiwan University, Taipei, Taiwan. His areas of interest are in computer relaying, bio-mechatronics, bio-effects of electromagnetic wave, sensing network, and neuro-engineering.

Chih-Feng Chao received the B.S. degree in mechanical engineering from Chang Gang University, Taoyuan, Taiwan, ROC, and the M.S. degree in bioindustrial mechatronics engineering from National Taiwan University (NTU), Taipei, Taiwan, ROC, in 2003 and 2006. His research interests are in the area of biomedical engineering, biomedical signal processing, and pattern recognition.

Ming-Jang Chiu received his B. Med. degree in medicine in 1984 and Ph.D. degree in electrical engineering in 2000 both from National Taiwan University, Taipei, Taiwan; and became a board certificated neurologist in Taiwan in 1989. He is currently an attending neurologist in National Taiwan University Hospital specialized in the diagnosis and treatment of patients with dementia. He has been the standing board member of Taiwan Alzheimer's Disease Association and secretary general of Taiwan society of clinical neurophysiology both since 2004. Dr. Chiu's research interests include cognitive neuroscience, sleep medicine and clinical neurophysiology.

Ren-Guey Lee received the M.S. degree from Department of Electrical Engineering, National Chen Kung University (NCKU), Taiwan, in 1989, and the Ph.D. degree from Department of Electrical Engineering, National Taiwan
University (NTU), in 2000. Since 2002, he has been with the Department of Electronic Engineering, National Taipei University of Technology, where he is currently an Associate Professor. His research interests include medical informatics, telecare and mobile care system, and wireless sensor network for biomedical applications.

Chwan-Lu Tseng graduated from the National Taipei Institute of Technology in 1985 and received the M.S. and Ph.D. degrees from National Taiwan University in 1991 and 1995, respectively, both in electrical engineering. Since 2000, he has been an Associate Professor in the Department of Electrical Engineering, National Taipei University of Technology. His research interests include signal processing, automation, robust control theory and its applications. He is a member of the Phi Tau Phi Scholastic Honor Society at National Taiwan University.

Robert Lin was born in Changhwa, Taiwan, ROC, in 1957. He received the M.S. degree in electronic engineering from Chung Yuan Christian University, Taiwan, ROC, in 2001. He is currently working toward his Ph.D. degree in Bio-industrial Mechatronics Engineering Department of National Taiwan University. He is currently a Senior Lecturer in Electrical Engineering Department of Lunghwa University of Science and Technology. At present, his interested research includes mix-mode IC design of VLSI, and the application of medical engineering. 